Short Research Communication

\title{
Senescence Affects Endothelial Cells Susceptibility to Dengue Virus Infection
}

\author{
Sazaly AbuBakar ${ }^{1 凶}$, Meng-Hooi Shu ${ }^{1}$, Jefree Johari ${ }^{1}$ and Pooi-Fong Wong ${ }^{\circledR}{ }^{凶}$ \\ 1. Tropical Infectious Diseases Research and Education Centre (TIDREC), Department of Medical Microbiology, Faculty of Medicine, \\ University of Malaya, 50603 Kuala Lumpur, Malaysia; \\ 2. Department of Pharmacology, Faculty of Medicine, University of Malaya, 50603 Kuala Lumpur, Malaysia.
}

$\triangle$ Corresponding author: Sazaly AbuBakar; Tel: +603 7967 5757; Fax: +603 7967 5757; E-mail: sazaly@um.edu.my.

(c) Ivyspring International Publisher. This is an open-access article distributed under the terms of the Creative Commons License (http://creativecommons.org/ licenses/by-nc-nd/3.0/). Reproduction is permitted for personal, noncommercial use, provided that the article is in whole, unmodified, and properly cited.

Received: 2013.10.15; Accepted: 2014.01.06; Published: 2014.04.04

\begin{abstract}
Alteration in the endothelium leading to increased vascular permeability contributes to plasma leakage seen in dengue hemorrhagic fever (DHF) and dengue shock syndrome (DSS). An earlier study showed that senescent endothelial cells (ECs) altered the ECs permeability. Here we investigated the susceptibility of senescing human umbilical vein endothelial cells (HUVECs) to dengue virus infection and determined if dengue virus infection induces HUVECs senescence. Our results suggest that DENV type-2 (DENV-2) foci forming unit (FFU) and extracellular virus RNA copy number were reduced by at least $35 \%$ and $85 \%$ in infection of the intermediate young and early senescent HUVECs, respectively, in comparison to infection of young HUVECs. No to low infectivity was recovered from infection of late senescent HUVECs. DENV infection also increases the percentage of HUVECs expressing senescence-associated (SA)- $\beta$-gal, cells arrested at the G2/M phase or 4N DNA content stage and cells with enlarged morphology, indicative of senescing cells. Alteration of HUVECs morphology was recorded using impedance-based real-time cell analysis system following DENV-2 infection. These results suggest that senescing HUVECs do not support DENV infection and DENV infection induces HUVECs senescence. The finding highlights the possible role of induction of senescence in DENV infection of the endothelial cells.
\end{abstract}

Key words: HUVECs, Endothelial cells; Senescence; Dengue virus, Endothelium permeability.

\section{Introduction}

Dengue is a zoonotic mosquito-borne disease endemic in most countries in the Southeast Asia (SEA), Latin America and the island nations of the Pacific [1-3]. World Health Organization (WHO) estimated that dengue is now present in over 125 countries with over 3.0 billion people at risk of getting infected and over 50-100 million new dengue cases are reported annually [4,5]. Dengue could present as mild dengue fever (DF) to severe dengue hemorrhagic fever (DHF) and dengue shock syndrome (DSS) [6]. In severe dengue, patients normally present with evidence of vascular plasma leakage which include raise in the hematocrit and present with ascites and pleural effusion [7]. Increase in the microvascular permeability is a possible mechanism contributing to the plasma leakage $[8,9]$. And this could be contributed by immune responses mount in response to the infection or as a result of direct infection of the endothelium [10-12]. Evidences suggesting the later have been reported in patients' autopsy materials and in animal models [13-17]. A number of cytokines and chemokines either secreted by the immune cells in response to the infection or by the infected endothelial cells (ECs) have also been identified [11, 12, 18-20]. It is suggested that these cytokines and chemokines act on the ECs affecting the gap junction resulting in transient increase in the endothelium permeability.

In addition, the potential involvement of DENV induced apoptosis has also been proposed to accommodate the finding that no gross morphological 
changes is seen in the endothelium of autopsy materials of patients who succumbed to DHF/DSS [11, 12, 21]. Currently, there is as yet only limited evidence to support induction of apoptosis in DENV infection of the relevant human ECs as the mechanism that triggers endothelium permeability. Alternatively, a recent study showed that the presence of senescing ECs alters the permeability of young ECs monolayer [22]. In relation, a number of earlier studies also showed induction of cell maturation following DENV infection $[13,23,24]$. Here we used the well-established ECs model culture system, human umbilical vein endothelial cells (HUVECs), to determine if DENV can productively infect senescing ECs and if the infection induces ECs senescence.

\section{Methods}

\section{Cell cultures}

Aedes albopictus mosquito cell line (C6/36) maintained in the Department of Medical Microbiology, Faculty of Medicine, University of Malaya were used. Cells were maintained in Eagle's minimum essential medium (EMEM) (supplemented with 1\% 10 $\mathrm{mM}$ non-essential amino acid and $1 \% \quad 10 \mathrm{mM}$ L-glutamine) with $10 \%$ heat-inactivated fetal bovine serum (FBS) (Hyclone, Thermo Fisher Scientific, USA) at $28^{\circ} \mathrm{C}$ in $3 \% \mathrm{CO}_{2}$.

HUVECs (ScienCell Research Laboratories, USA) were serially passaged in endothelial cell medium (ECM) (supplemented with $1 \%$ endothelial cell growth supplement and $1 \% 10,000 \mathrm{U} / \mathrm{ml} / 10,000$ $\mu \mathrm{g} / \mathrm{ml}$ penicillin/streptomycin) with $5 \%$ FBS (ScienCell Research Laboratories, USA) at $37^{\circ} \mathrm{C}$ in a humidified incubator with $5 \% \mathrm{CO}_{2}$ until they reached replicative senescence as previously described [25]. At each passage, HUVECs were staged using senescence-associated (SA)- $\beta$-galactosidase $(\beta$-gal) staining and cell cycle analysis.

\section{DENV infection of HUVECs}

HUVECs at young, intermediate young, early senescent and late senescent were seeded into 24-well cell culture plates (Falcon, BD Biosciences, USA) at a density of $5 \times 10^{4}$ cells/well. The following day, the cell culture medium was removed and passage 3 clinical isolate of DENV type-2 (DENV-2) strain MY91-99133 (Department of Medical Microbiology, University Malaya Medical Centre Virology repository) was added to give an estimated multiplicity of infection (MOI) of 10 per cell. Cells were incubated with the inoculum for 1 hour at room temperature with constant gentle agitation to allow virus adsorption. After an hour, the inoculum was removed and fresh ECM with 2\% FBS was added. Cells were cul- tured for 7 days at $37^{\circ} \mathrm{C}$ in a humidified incubator with $5 \% \mathrm{CO}_{2}$. The cell culture supernatant was centrifuged and used for virus infectivity or replication assay by focus-forming assay and quantitative real-time (RT)-polymerase chain reaction (PCR). The remaining cell monolayers were trypsinized and stained for $\beta$-gal staining and cell cycle analysis.

\section{Focus-forming assay}

Focus-forming assay was performed to titrate DENV-2 using the C6/36 cells as previously described by Okuno et al. [26] and Zandi et al. [27].

\section{Quantitative RT-PCR}

Quantitative RT-PCR amplification was performed to quantitate DENV-2 genomic RNA copy number using a customized one-step TaqMan real-time RT-PCR (Applied Biosystems, USA) following the manufacturer's recommended protocol.

\section{SA- $\beta$-gal staining}

In situ staining for SA- $\beta$-gal was performed following the manufacturer's recommended protocol (Cell Signaling Technology, USA). Stained cells were viewed and counted at $80 \mathrm{x}$ magnification under brightfield using a stereomicroscope (Nikon, Japan). The proportion of SA- $\beta$-gal positive cells which stained as blue-turquoise precipitate in cells was calculated by dividing with the total number of cells counted per microscopic field. At least 10 microscopic fields were used per datum point.

\section{Cell cycle analysis}

Cell cycle analysis was performed by measuring the DNA content using the CycleTEST TM Plus DNA reagent kit (BD Biosciences, USA) following the manufacturer's recommended protocol. The DNA content of 15, 000 cells were measured with fluorescence-activated cell sorting (FACS) CANTO ${ }^{\mathrm{TM}}$ II flow cytometer (BD Biosciences, USA). All measurements were performed under identical instrument settings. The relative percentages of cells in G0/G1, S and G2/M population of the cell cycle were estimated from the propidium iodide (PI) histograms and analyzed using the MODFIT software (BD Biosciences, USA). Cells arrested in the G2/M phase were identified by the $4 \mathrm{~N}$ DNA content peak which could also reflect the accumulation of cells in the G1 phase following polyploidization [28].

\section{Real time growth kinetic analysis}

Real time growth kinetics of HUVECs following DENV infection was monitored using the impedance-based Real-Time Cell Analysis (RTCA) system (ACEA Biosciences, USA). A method modified from Lee et al. [29] was used. Briefly, ECM with 2\% FBS was 
added to 96-well E-plate (ACEA Biosciences, USA) and used as the background reading. HUVECs at young, intermediate young and early senescent were seeded into each well at a density of $5 \times 10^{3}$ cells/well. Cells proliferation and morphological changes were continuously monitored and recorded at every 5 minutes intervals. When the cell culture entered logarithmic growth phase, the culture medium was removed and DENV-2 inoculum was added. Cells were infected for 1 hour, removed and ECM was added. Cells were continuously monitored at every 5 minutes intervals for up to 120 hours. Cell growth impedance readings were expressed as cell index [30]. Growth curves were normalized to the cell index obtained at the last measured time point before infection.

\section{Statistical analysis}

The data for focus-forming assay, quantitative RT-PCR, SA- $\beta$-gal staining and real time growth kinetic analysis were expressed as median $(25 \%$ and $75 \%$ quartile) from four independent experiments, mean \pm S.D. from two independent experiments, mean \pm standard deviation (S.D.) with $95 \%$ confidence interval $(95 \% \mathrm{CI})$ from two independent experiments and mean \pm S.D. from two independent experiments, respectively. This data were subjected to one-way analysis of variance (ANOVA) and Bonferroni's multiple comparison test. $p<0.05$ was taken as statistically significant. For data obtained from cell cycle analysis, we used two-way ANOVA and Bonferroni's posttest. This data were expressed as mean \pm S.D. from two independent experiments. Statistical significance was taken when $p<0.05$. All statistical analyses were done using GraphPad Prism 4.0 Software (USA).

\section{Results and Discussion}

The pathogenesis of DHF/DSS in dengue disease is still not well understood. Increased in the vascular permeability resulting in plasma leakage has been suggested as possible mechanisms contributing to DHF/DSS. Since ECs dysfunction leads to increase in vascular permeability, many earlier studies have used the in vitro cultured ECs as a model cell culture system for the study of the effects and permissiveness of dengue virus infection [8, 18, 19, 31-35]. A recent study exhibited that the presence of senescing ECs resulted in decreased endothelial barrier function of the non-senescent monolayer ECs [22]. Here we used the similar well-established model to first examine if DENV can productively infect senescing ECs and secondly to determine if the infection induces ECs senescence.

Continuous serial passaging of HUVECs led to exhaustion of their proliferative capacity. Young HUVECs [6-7 passages and $\sim 5-10$ population dou- blings (PD)] usually reached monolayer within 3 days when maintained in $75 \mathrm{~cm}^{2}$ cell culture flask. At this stage less than $10 \%$ cells stained positive for SA- $\beta$-gal (Fig. 1A: top left panel; Fig. 1C). The SA- $\beta$-gal activity has been extensively used as a biomarker for staging senescence [36]. These percentages gradually increase for intermediate young (16-17 passages and 33-36 PD; $13.60 \pm 4.31 \%$ ), early senescent (24-25 passages and $\sim 45-48 \mathrm{PD} ; 49.12 \pm 9.53 \%$ ) and late senescent HUVECs (26 passages onwards and about 49-50 PD; $78.25 \pm 5.94 \%$ ) (Fig. 1A: second, third and bottom left panel; Fig. 1C). The final senescent stage was characterized by irreversible growth arrest, morphologically enlarged cells and increased in the nuclei size. At this stage more than $75 \%$ cells stained positive for SA- $\beta$-gal (Fig. 1A: bottom left panel; Fig. 1C) and more than $90 \%$ cells were at the $\mathrm{G} 2 / \mathrm{M}$ phase or $4 \mathrm{~N}$ DNA content stage (Fig. 1B: bottom left panel) [28, 36]. There was a direct correlation between the number of cells expressing SA- $\beta$-gal activity, cells arrested in G2/M phase and the HUVECs passage number. The percentages of cells at G0/G1, S and G2/M phases of the cell cycle staining was determined to further verify results obtained using the SA- $\beta$-gal staining.

In the present study, DENV-2 infectivity or replication in HUVECs, at each passage number was determined by focus-forming assay and quantitative RT-PCR. Focus-forming assay showed that DENV titer of $550.00(450.00,850.00) \mathrm{ffu} \mathrm{ml}^{-1}$ was obtained for infection of young HUVECs (Table 1). A titer of 250.00 (150.00, 600.00) ffu ml-1 and $35(10.00,77.50) \mathrm{ffu} \mathrm{ml}^{-1}$ were obtained for infection of intermediate young and early senescent HUVECs, respectively. No infectivity was recovered from infection of late senescent HUVECs. Mock-infected HUVECs were used as control and no virus foci were recovered. Quantitative RT-PCR amplification showed that the amount of extracellular dengue virus specific RNA peaked in young HUVECs infection at 1, 220.25 \pm 372.83 copies of RNA and decreased gradually in intermediate young to $787.67 \pm 410.85$ copies of RNA, early senescent to $183.13 \pm 92.60$ copies of RNA and late senescent HUVECs to $60.50 \pm 26.16$ copies of RNA, respectively (Table 1). Aged-dependent decrease in DENV-2 infectivity or replication in HUVECs was observed using ffu and quantitative RT-PCR. These results suggested that clinical isolate of DENV-2 can establish productive infection of young, intermediate young and early senescent HUVECs. This is consistent with most of the earlier reports suggesting possible direct DENV infection of HUVECs [8, 18, 19, 31-35]. The infectivity, however, is very much dependent on cell age, with infection of young cells resulted in the highest number of infectious virus recovered. Low or no infectious virus was recovered from the infection 
of late senescent HUVECs, suggesting that late senescent cells did not support productive DENV infection. It is noted here that most of the earlier work with infection of ECs did not take into consideration the specific age of cells used for infection.

Table I. DENV-2 infection of each passage of HUVECs. Titer of DENV-2 and genomic virus load expressed as median (25\% and $75 \%$ quartile) from four independent experiments and mean \pm S.D. from two independent experiments, respectively.

\begin{tabular}{lll}
\hline HUVECs & Virus titer count $\left(\mathrm{ffu} \mathrm{ml}^{-1}\right)$ & $\begin{array}{l}\text { Virus load (copies of } \\
\text { RNA) }\end{array}$ \\
\hline Young & $550.00(450.00,850.00)^{*}$ & $1,220.25 \pm 372.83$ \\
Intermediate young & $250.00(150.00,600.00) *$ & $787.67 \pm 410.85$ \\
Early senescent & $35.00(10.00,77.50)^{*}$ & $183.13 \pm 92.60 *$ \\
Late senescent & $0.00 \pm 0.00 *$ & $60.50 \pm 26.16^{*}$ \\
\hline ' $*$ ' $^{*}$ showed significance difference in comparison to young HUVECs value: $p<0.05$.
\end{tabular}

In a recent study, Krouwer et al. [22] showed that the presence of senescent cells induces alteration of the non-senescent ECs resulting in increased in cells permeability. Here we examined the possibility of DENV infection itself induced senescence of HUVECs. Initially, the percentage of senescent HUVECs was determined by the SA- $\beta$-gal staining. Results from representative experiment suggest that mockand DENV-2-infected young HUVECs showed $5.77 \pm$ $3.18 \%$ [95\% confidence interval $(\mathrm{CI})=4.28-7.26]$ and $8.86 \pm 2.15 \%(95 \% \mathrm{CI}=7.85-9.86)$ SA- $\beta$-gal positive cells, respectively (Fig. 1A: top left and right panel; Fig. 1C). $13.60 \pm 4.31 \%$ (95\% CI = 11.58-15.61), $49.12 \pm$ 9.53\% (95\% CI $=44.66-53.58)$ and $78.25 \pm 5.94 \%(95 \%$ $\mathrm{CI}=74.00-82.50)$ SA- $\beta$-gal positive cells were observed for the intermediate young, early senescent and late senescent mock-infected HUVECs, respectively (Fig. 1A: second, third and bottom left panel; Fig. 1C). There were $\sim 2.41$ times and $\sim 1.42$ times increment in the SA- $\beta$-gal positive cells when HUVECs at intermediate young and early senescent were infected with DENV-2, in comparison to the mock-infected control (Fig. 1A: second and third panel; Fig. 1C). These results suggested that significantly $(p<0.001)$ increasing number of senescent cells exhibiting SA- $\beta$-gal activity were observed in DENV-2-infected HUVECs cell cultures.

The effects of DENV infection on HUVECs senescence were also determined using the flow cytometry for cells arrested at the different cell cycle phases. Results from one representative study shown here, suggest that approximately $67.10 \pm 1.58 \%$ and $63.11 \pm 4.07 \%$ of HUVECs mock- and DENV-2-infected, respectively, were at the G0/G1 phase or 2N DNA content stage for infection of young cells (Fig. 1B: top left and right panel). Only, $12.55 \pm$ $2.16 \%$ and $15.95 \pm 7.13 \%$ of the young HUVECs mock- and DENV-2-infected, respectively, were at the G2/M phase or $4 \mathrm{~N}$ DNA content stage. Infection at the intermediate young passage, HUVECs mock- and DENV-2-infected showed $77.49 \pm 3.66 \%$ and $85.37 \pm$ $0.73 \%$ cells, respectively, at the G0/G1 phase with concomitant decrease of the percentage of cells at G2/M phase to background level, $3.09 \pm 4.37 \%$ and $4.23 \pm 0.69 \%$, respectively (Fig. 1B: second left and right panel). Approximately $53.68 \pm 24.96 \%$ of HUVECs at early senescent were arrested in the G2/M phase when cells were infected with DENV-2 (Fig. 1B: third right panel). In comparison, only $28.25 \pm 17.32 \%$ of the mock-infected cell culture was at G2/M phase (Fig. 1B: third left panel). Approximately $94.78 \%$ of the mock-infected HUVECs were arrested in the G2/M phase with a concomitant reduction of the G0/G1 phase to background level when infection was done at late senescent stage (Fig. 1B: bottom left panel). These results suggested that DENV-2-infection of HUVECs resulted in increase in the number of cells arrested at the G2/M phase or 4 N DNA content stage. The findings were consistent with the earlier finding that DENV infection increased the number of cells stained positive for SA- $\beta$-gal activity, a marker for senescent endothelial cells [37].

A recent study showed that senescing ECs disrupted cell-cell junctions and increased the ECs monolayer permeability [22]. Here, we utilized the impedance-based RTCA system to monitor HUVECs following DENV infection. Significant changes in cells indices would suggest significant cells proliferation and morphological changes have occurred. Infected HUVECs morphological changes determined by RTCA occurred as early as at 8 hours post-infection. Area under the curve for the mock- and DENV-2-infected young HUVECs was $185.03 \pm 24.28$ and $206.00 \pm 10.38$, respectively (Fig. 2A; Table 2). The area under the curve was $286.80 \pm 9.68$ for the intermediate young DENV-2-infected HUVECs, in comparison to the mock-infected which was $199.37 \pm 28.56$ (Fig. 2B; Table 2). Whereas, the area under the curve for DENV-2-infected early senescent HUVECs was $257.35 \pm 149.83$, in comparison to the mock-infected with $230.42 \pm 149.64$ (Fig. 2C; Table 2). Normalized area under the curve for intermediate young HUVECs (area under the curve for DENV-2-infected minus area under the curve for mock-infected) was 87.43 , in comparison to 20.97 for young and 26.93 for early senescent HUVECs (Table 2). The normalized cell index increased with increasing cell passage number (Fig. 2), suggesting cell morphological changes including cell enlargement occurred for intermediate young HUVECs infected with DENV-2 (Fig. 2B). Late senescent cells were not used in this part of the study as these cells were no longer responsive to further 
stimulation or infection. For infection of the intermediate young HUVECs, the changes coincided with the point during which over $85 \%$ of the infected cells were in the G0/G1 phase, suggesting that the morphologic changes is associated with the cell cycle stage (Fig. 1B: second right panel).

$\mathbf{A}$

Mock-infected
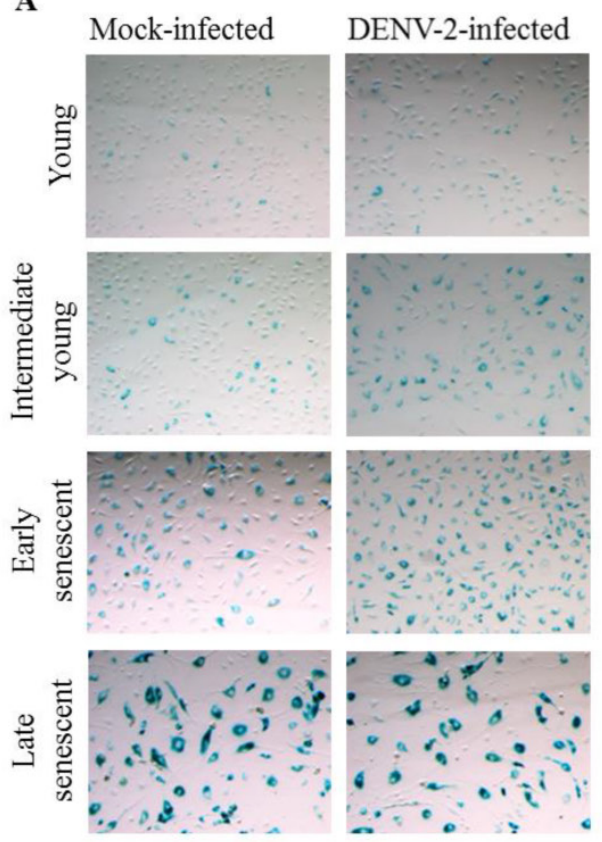

C

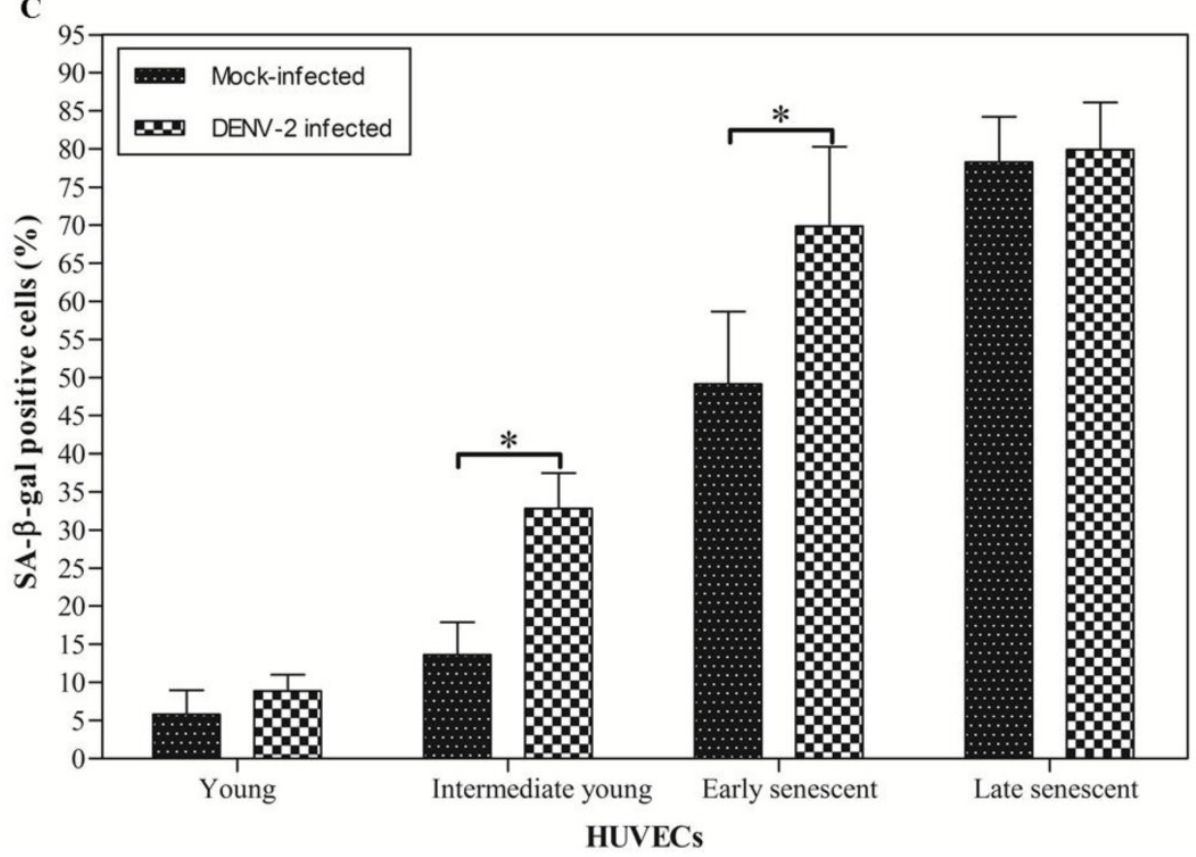
mean \pm S.D. from two independent experiments. minus area under the curve for mock-infected.

B
Mock-infected
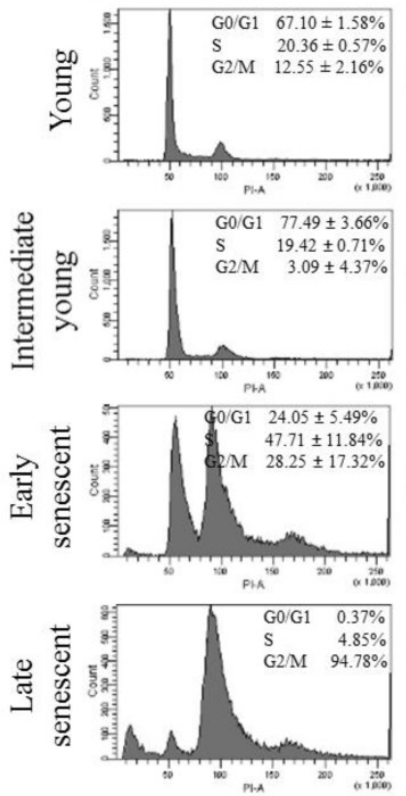

HUVECs

Table 2. Real-time growth kinetic analysis of both mock- and DENV-2-infected HUVECs at each passage number. Data are expressed as

\begin{tabular}{|c|c|c|c|}
\hline \multirow[t]{2}{*}{ HUVECs } & \multicolumn{2}{|c|}{ Area Under the Curve } & \multirow{2}{*}{$\begin{array}{l}\text { "Normalised Area } \\
\text { Under the Curve }\end{array}$} \\
\hline & Mock-infected & DENV-2 Infected & \\
\hline Young & $185.03 \pm 24.28$ & $206.00 \pm 10.38$ & 20.97 \\
\hline Intermediate young & $199.37 \pm 28.56$ & $286.80 \pm 9.68$ & 87.43 \\
\hline Early senescent & $230.42 \pm 149.64$ & $257.35 \pm 149.83$ & 26.93 \\
\hline
\end{tabular}

The larger the area under the curve, suggest larger cell morphology changes.

- Normalised area under the curve is area under the curve for DENV-2-infected

DENV-2-infected
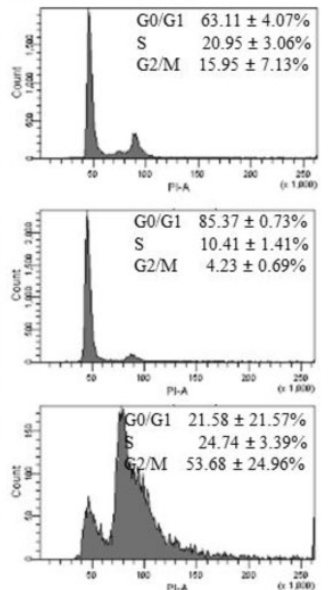

Figure I. SA- $\beta$-gal staining and cell cycle population distribution of mock- and DENV-2-infected HUVECs at each passage number. (A) SA- $\beta$-gal positive cells which stained as blue-turquoise precipitate in cells. (B) DNA content in I5, 000 cells at the different phase during doublings. The relative percentages of cells in G0/GI, $S$ and G2/M population of the cell cycle were estimated from the propidium iodide (PI) histograms. (C) The percentage of the SA- $\beta$-gal positive cells. The data shown are mean \pm S.D. from two independent experiments with '*', $p<0.00$ I represent significance difference. 

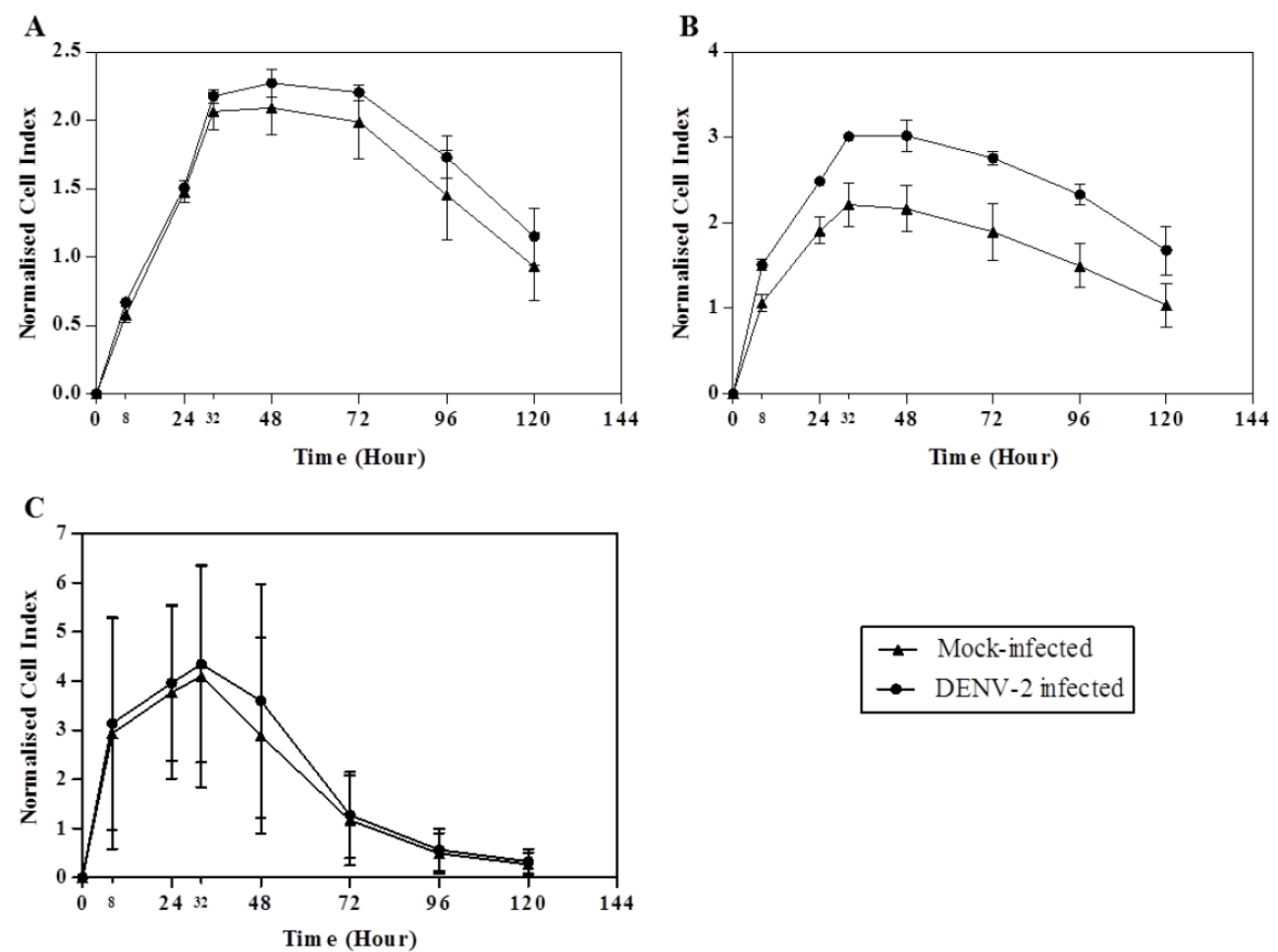

Figure 2. Dynamic assessment of HUVECs proliferation and morphological changes of both mock- and DENV-2-infected at each passage number. Growth kinetics of (A) young, (B) intermediate young and (C) early senescent HUVECs which captures proliferation, morphological changes and induction of apoptosis events was measured. The data for cell index was expressed as mean \pm S.D. from two independent experiments.

Earlier studies have shown that DENV infection of HUVECs stimulated the expression of stress-, defense-, wound-, inflammation- and signaling-related genes whilst down-regulating those associated with membrane-, cytoskeleton- and vascular endothelial cadherin-related genes [12, 18, 31, 35]. Upregulation and downregulation of these genes are thought to concertedly contribute to the ECs morphologic changes leading to increased permeability of the endothelium. Here, we showed using the sensitive RTCA method that HUVECs morphological changes occurred following infection with DENV and these changes correlated with the induction of HUVECs senescence.

\section{Conclusions}

Herein, we propose that dengue virus infection induces ECs to undergo senescent state. Since DENV could not establish productive infection in senescent cells, we suggest that the induction of senescence could be the HUVECs defense mechanism against the virus insult. Though an earlier study has shown that senescing cells induced ECs permeability [22], suggesting possible role of dengue virus-induced senescing cells in induction of vascular endothelial leakage, further studies will be needed to verify this possibility.

\section{Acknowledgements}

This study was supported by the Ministry of Higher Education (MOHE), Malaysia under the Long Term Research Grant Scheme (LRGS/2011A/UM/ Penyakit-Berjangkit) and High Impact Research (HIR)-MOHE Grant (E000013-20001).

\section{Competing Interests}

The authors have declared that no competing interest exists.

\section{References}

1. AbuBakar S, Wong PF, Chan YF. Emergence of dengue virus type 4 genotype IIA in Malaysia. Journal of General Virology. 2002; 83: 2437-2442.

2. Shafee N, AbuBakar S. Dengue virus type 2 NS3 protease and NS2B-NS3 protease precursor induce apoptosis. Journal of General Virology. 2003; 84:2191-2195.

3. Nathan MB, Dayal-Drager R. Recent epidemiological trends, the global strategy and public health advances in dengue. Report on Dengue. 2006;: 30-34.

4. [Internet] World Health Organization. Dengue and severe dengue. http://www.who.int/mediacentre/factsheets/fs117/en/index.html.

5. [Internet] World Health Organization. Global strategy for dengue prevention $\begin{array}{ll}\text { and control 2012-2020. } & \text {. }\end{array}$ http://apps.who.int/iris/bitstream/10665/75303/1/9789241504034_eng.pdf.

6. World Health Organization Regional Office for South-East Asia. Comprehensive guidelines for prevention and control of dengue and dengue haemorrhagic fever. World Health Organization. 2011.

7. Special Programme for Research, Training in Tropical Diseases, World Health Organization; Department of Control of Neglected Tropical Diseases, World Health Organization; Department of Epidemic and Pandemic Alert. Dengue: guidelines for diagnosis, treatment, prevention and control. World Health Organization. 2009.

8. Liu P, Woda M, Ennis FA, et al. Dengue virus infection differentially regulates endothelial barrier function over time through type I interferon effects. The Journal of Infectious Diseases. 2009; 200: 191-201. 
9. Srichaikul T, Nimmannitya S. Haematology in dengue and dengue haemorrhagic fever. Baillière's Clinical Haematology. 2000; 13(2): 261-276.

10. Lei HY, Huang KJ, Lin YS, et al. Immunopathogenesis of dengue hemorrhagic fever. American Journal of Infectious Diseases. 2008; 4(1): 1-9.

11. Basu A, Chaturvedi UC. Vascular endothelium: the battlefield of dengue viruses. FEMS Immunology and Medical Microbiology. 2008; 53: 287-299.

12. Srikiatkhachorn A. Plasma leakage in dengue haemorrhagic fever. Thrombosis and Haemostasis. 2009; 102(6): 1042-1049.

13. Noisakran S, Onlamoon N, Songprakhon $\mathrm{P}$, et al. Cells in dengue virus infection in vivo. Advances in Virology. 2010.

14. McBride WJH, Bielefeldt-Ohmann H. Dengue viral infections: pathogenesis and epidemiology. Microbes and Infection. 2000; 2(9): 1041-1050.

15. Dalrymple NA, Mackow ER. Endothelial cells elicit immune-enhancing responses to dengue virus infection. Joural of Virology. 2012; 86(12): 6408-6415.

16. Mota J, Rico-Hesse R. Dengue virus tropism in humanized mice recapitulates human dengue fever. Plos One. 2011; 6(6): e20762.

17. Kyle JL, Beatty PR, Harris E. Dengue virus infects macrophages and dendritic cells in a mouse model of infection. The Journal of Infectious Diseases. 2007; 195: 1808-1817.

18. Warke RV, Xhaja K, Martin KJ, et al. Dengue virus induces novel changes in gene expression of human umbilical vein endothelial cells. Journal of Virology. 2003; 77(21): 11822-11832.

19. Avirutnan P, Malasit P, Seliger B, et al. Dengue virus infection of human endothelial cells leads to chemokine production, complement activation and apoptosis. The Journal of Immunology. 1998; 161: 6338-6346.

20. Appanna R, Wang SM, Ponnampalavanar SA, et al. Cytokine factors present in dengue patient sera induces alterations of junctional proteins in human endothelial cells. The American Journal of Tropical Medicine and Hygiene. 2012; 87(5): 936-942

21. Limonta D, Capó V, Torres G, et al. Apoptosis in tissues from fatal dengue shock syndrome. Journal of Clinical Virology. 2007; 40: 50-54.

22. Krouwer VJD, Hekking LHP, Langelaar-Makkinje M, et al. Endothelial cell senescence is associated with disrupted cell-cell junctions and increased monolayer permeability. Vascular Cell. 2012; 4(12): 1-10.

23. Azeredo EL, Oliveira-Pinto LMD, Zagne SM, et al. NK cells, displaying early activation, cytotoxicity and adhesion molecules, are associated with mild dengue disease. Clinical and Experimental Immunology. 2006; 143(2): 345-356.

24. Nightingale ZD, Patkar C, Rothman AL. Viral replication and paracrine effects result in distinct, functional responses of dendritic cells following infection with dengue 2 virus. Journal of Leukocyte Biology. 2008; 84(4): 1028-1038.

25. Van der Loo B, Fenton MJ, Erusalimsky JD. Cytochemical detection of a senescence-associated $\beta$-galactosidase in endothelial and smooth muscle cells from human and rabbit blood vessels. Experimental Cell Research. 1998; 241:309-315

26. Okuno Y, Fukunaga T, Srisupaluck S, et al. A modified PAP (peroxidase-anti-peroxidase) staining technique using sera from patients with dengue hemorrhagic fever (DHF): 4 step PAP staining technique. Biken Journal. 1979; 22(4): 131-135.

27. Zandi K, Teoh BT, Sam SS, et al. In vitro antiviral activity of Fisetin, Rutin and Naringenin against dengue virus type-2. Journal of Medicinal Plants Research. 2011; 5(23): 5534-5539.

28. Wagner M, Hampel B, Bernhard D, et al. Replicative senescence of human endothelial cells in vitro involves G1 arrest, polyploidization and senescence-associated apoptosis. Experimental Gerontology. 2001; 36: 1327-1347.

29. Lee ST, Wong PF, Cheah SC, et al. Alpha-tomatine induces apoptosis and inhibits nuclear factor-kappa $\mathrm{B}$ activation on human prostatic adenocarcinoma PC-3 cells. Plos One. 2011; 6(4): e18915

30. Solly K, Wang X, Xu X, et al. Application of Real-Time Cell Electronic Sensing (RT-CES) technology to cell-based assays. Assay and Drug Development Technologies. 2004; 2(4): 363-372.

31. Huang YH, Lei HY, Liu HS, et al. Dengue virus infects human endothelial cells and induces IL-6 and IL-8 production. The American Journal of Tropical Medicine and Hygiene. 2000; 63: 71-75.

32. Huang YH, Lei HY, Liu HS, et al. Tissue plasminogen activator induced by dengue virus infection of human endothelial cells. Journal of Medical Virology. 2003; 70: 610-616.

33. Dalrymple N, Mackow ER. Productive dengue virus infection of human endothelial cells is directed by heparan sulfate-containing proteoglycan receptors. Journal of Virology. 2011; 85(18): 9478-9485.

34. Jacobs M, Levin M. An improved endothelial barrier model to investigate dengue haemorrhagic fever. Journal of Virological Methods. 2002; 104: 173-185.

35. Dewi BE, Takasaki T, Kurane I. Peripheral blood mononuclear cells increase the permeability of dengue virus-infected endothelial cells in association with downregulation of vascular endothelial cadherin. Journal of General Virology. 2008; 89: 642-652.

36. Heo JI, Cho JH, Kim JR. HJURP regulates cellular senescence in human fibroblasts and endothelial cells via a p53-dependent pathway. The Journals of Gerontology Series A: Biological Sciences and Medical Sciences. 2013.

37. Dimri GP, Lee $\mathrm{XH}$, Basile G, et al. A biomarker that identifies senescent human cells in culture and in aging skin in vivo. Proceedings of the National Academy Sciences of the United States of America. 1995; 92: 9363-9367. 